\title{
Hydrocephalus in Africa: A surgical perspective
}

\begin{abstract}
N. Bauman, MD, Senior Surgical Resident, McMaster University Faculty of Medicine, Hamilton, ON, Canada and D. Poenaru, MD, MHPE, FRCSC, FACS, FICS, FCS-ECSA, Adjunct Professor of Surgery and Paediatrics, Queens' University, Kingston, Canada, Hon. Professor of Surgery, Aga Khan University, Nairobi, Kenya, Medical Education and Research Director, AIC Kijabe Hospital, Kijabe, P.O. Box 20, Kijabe 00220, Kenya
\end{abstract}

Correspondence and reprints request to:

Prof. D. Poenaru, P.O. Box 20, Kijabe 00220, Kenya, Email: MedEdDirokh@kijabe.net

\section{Introduction}

Hydrocephalus is the excessive accumulation of cerebrospinal fluid (CSF) within the cranial vault due to excessive production or inadequate absorption. The management of hydrocephalus in developing nations is hindered by significant economic constraints and delays in treatment - most patients in fact do not present for at least seven months after the onset of clinical symptoms (1). CSF shunts are expensive and not always available, but can be used to treat hydrocephalus regardless of etiology. The management of hydrocephalus and the complications associated with its treatment require considerable surgical judgment and a life-long approach to patient follow-up.

\section{Demographics}

The prevalence of hydrocephalus in developed nations is estimated as $0.9-1.2 / 1000$ (2). No reliable estimate is available in the African literature, but its incidence is likely higher because of untreated/poorly treated neonatal meningitis and nutritional deficiencies.

\section{Aetiology \& pathophysiology}

Cerebrospinal fluid (CSF) is produced predominantly by the choroid plexus of the four cerebral ventricles, at a rate of $20 \mathrm{ml} /$ hour. It flows via the foramina of Luschka and Magendie into the subarachnoid space, and is absorbed by the arachnoid villi into the venous system via the superior saggital sinus. Hydrocephalus has been categorized as communicating or noncommunicating: the former is due to failure of CSF absorption by the arachnoid villi, whereas the latter involves obstruction of CSF flow into the subarachnoid space. A small minority of cases exhibit excessive production of CSF most commonly secondary to a choroid plexus papilloma.

Figure 1: Aetiology of hydrocephalus in Uganda

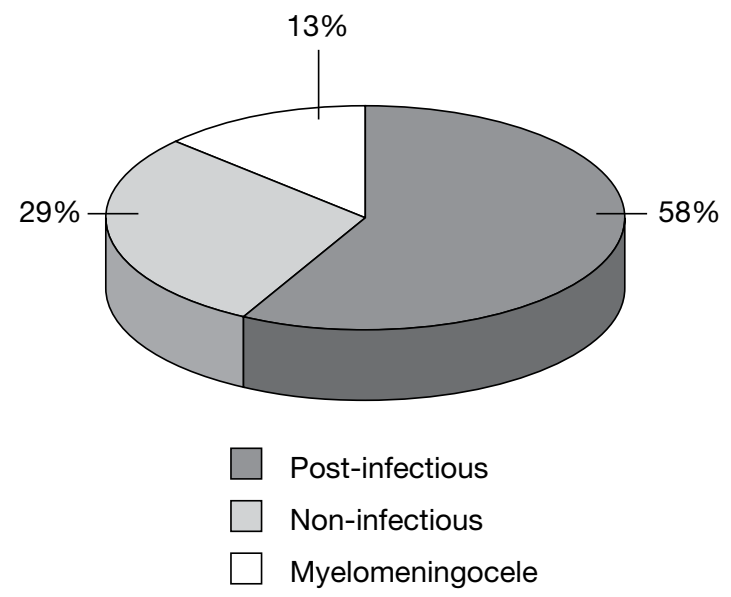

In developed nations, hydrocephalus has historically been most commonly due to myelomeningocele, with the post-hemorrhagic hydrocephalus of prematurity becoming at least as common in recent years (2). Some reports have suggested that in central Africa the most common causes of hydrocephalus are - neural tube defects and congenital aqueductal stenosis (3). Similarly, 
in Zambia, the ratio of congenital to "postmeningitic" hydrocephalus has been reported to be 2:1 (4). In contrast, a well-documented prospective series in East Africa has shown the etiology of hydrocephalus to be $57 \%$ post-infectious, $29 \%$ non post-infectious, and myelomeningocele (Figure 1). Thus, neonatal meningitis /ventriculitis is likely the most common cause of hydrocephalus in East Africa (1).

Despite the prevalence of malaria in Africa and case reports of associated hydrocephalus, there is no clear relationship between its cerebral form and subsequent hydrocephalus (5). On the other hand the pathophysiology of hydrocephalus in the setting of myelomeningocele is multifactorial: it may be secondary to obstruction at the aqueduct, ventricular outlet, craniocervical junction, or arachnoid granulations.

\section{Clinical presentation}

The clinical presentation of hydrocephalus is characterized by signs and symptoms of increased intracranial pressure (ICP). Symptoms may include: headache, vomiting, failing vision, drowsiness, fatigue, deteriorating mental function, and enlarged head circumference. Signs include: wide tense fontanel, papilloedema, reduced visual acuity, failure of upward gaze (the sunsetting sign), general clumsiness, dyspraxic gait, and increasing head circumference. Older children will not present with increased head circumference, and often complain of the classic triad: headache, vomiting and lethargy (Table 1).

Table 1. Signs and symptoms of increased intracranial pressure

\begin{tabular}{lc} 
Acute & Chronic \\
Headache & Accelerated head \\
Nausea/Vomiting & growth \\
Lethargy & Loss of developmental \\
Papilloedema & milestones \\
CN IV palsy & Difficulty in school \\
Scalp vein distention & Vision loss \\
$\begin{array}{l}\text { Early morning headache } \\
\text { Headache exacerbated by }\end{array}$ & Gait disturbance \\
recumbency & \\
\hline
\end{tabular}

\section{Investigations}

Clinical examination is the most readily available investigation for the diagnosis of increased ICP, shunt malfunction or infection. All shunts should be examined and manipulated, although pumping of the shunt is not a reliable test of malfunction. Nevertheless, classic teaching suggests that a reservoir that is difficult to depress (or refills instantaneously) may indicate distal obstruction whereas slow filling of the reservoir may indicate obstruction proximally (6).

Cranial ultrasonography (US) is an essential diagnostic tool in developing countries: it can readily assess ventricular size with minimal training, and is relatively inexpensive. Depending on operator skill, the size of the fourth ventricle can be assessed on US as a proxy indication of the patency of the aqueduct. This may be particularly relevant in stratifying patients for treatment with prosthetic shunts versus endoscopic third ventriculostomy (ETV). Serial US imaging may be appropriate in patients with an equivocal presentation of increased ICP prior to subjecting them to shunt revision. All children with shunts should be followed up regularly, including baseline US within three months of surgery. Although acute changes from baseline may help in the subsequent diagnosis of shunt failure, up to a third of patients will not exhibit any evidence of ventriculomegaly (6).

Both computed tomography (CT) and magnetic resonance imaging (MRI) are excellent modalities, but their routine use is prohibited by cost in developing nations. Nevertheless, CT may be necessary in assessing the ventricular size in older children with closed fontanels noted. Evidence of increased ICP in children with closed fontanels can also be obtained through direct measurement of CSF pressure by lumbar puncture: the CSF column height is measured in a piece of intravenous (IV) tubing connected to the spinal needle via a 3-way stopcock.

Although rarely required, a "shuntogram" of the entire radiopaque shunt tract via a series of plain X-rays encompassing the skull, chest and abdomen is helpful when there is clinical suspicion of possible shunt migration or discontinuity (7). 
Documentation of shunt infection or ventriculitis may require culturing of CSF samples from the ventricles (in the neonate) or from the shunt reservoir itself. There is a small but real risk of CSF contamination with each diagnostic tap. A value of 50-100 white cells/ $\mathrm{mm}^{3}$ is considered indicative of an infected CSF, as is elevated protein, decreased sugar, and naturally the presence of bacteria on Gram stain. A bloody tap may erroneously elevate the white cell number. Any febrile child with a shunt should be examined for the possibility of other common febrile illnesses, especially if they are beyond 6 months post-shunt insertion. Malaria, otitis media, and viral gastroenteritis should be excluded, in addition to the possibility of urinary tract infection particularly in those with spina bifida and bladder dysfunction (6).

\section{Management}

The definitive management of hydrocephalus at present remains surgical. The diuretic acetazolamide has been shown to decrease CSF production in animal and human studies (8), but is of temporary benefit and should only be used in the palliative setting or in equivocal cases until a definitive diagnosis can be made. It has also been used in post-hemorrhagic hydrocephalus of the newborn as a temporizing maneuver to avoid shunting (9).

Ventriculo-peritoneal shunt: The most common surgical intervention to treat hydrocephalus is the insertion of a shunt through the skull and cortical mantle into the ventricle, with the distal catheter placed into a physiologic drainage basin, typically the peritoneal space (ventriculoperitoneal or VP shunt). Other sites for CSF diversion include the right atrium and the pleural space. The advantage of a CSF shunt is that it is beneficial in nearly all types of hydrocephalusregardless of etiology.

CSF shunts usually contain three parts: a ventricular catheter, a valve, and a distal catheter. Most valves are designed to allow for sampling via needle puncture. The so-called "differential pressure valves" use the gradient between the ventricle and the tip of the distal catheter to effect flow. "Medium pressure" valves are those which drain CSF if the pressure gradient is $>10 \mathrm{~mm} \mathrm{Hg}$, and are used most commonly. Although many different valve designs exist including "siphon limiting", "flow limiting", and "programmable" valves (whose settings can be changed using an external magnet) there is limited evidence for their benefit. A large randomized trial demonstrated no difference in time to first shunt failure in the treatment of children with newly diagnosed hydrocephalus when comparing a standard differential pressure valve compared to two other higher generation valves (10). Similarly, the use of an adjustable shunt was not shown to be of any benefit in terms of overall survival (11). Finally, and of most relevance to the developing world, there is good evidence from a prospective randomized controlled trial demonstrating that the Chhabra ${ }^{\circledR}$ shunt (made by Surgiwear in India) is equivalent to its common Western counterpart in incidence of shunt complications, despite it being almost $1 / 20$ the cost (12). The Chhabra shunts are made available for free to qualifying centres through the International Federation of Spina Bifida and Hydrocephalus (www.ifglobal.org). In extreme situations, a piece of IV tubing or a sterile Silastic ${ }^{\circledR}$ feeding catheter can be used as VP shunts, but these non-valved alternatives are associated with frequent complications and not recommended.

Alternative CSF drainage sites: Pleural shunts are rarely required, but can be placed via the 4th to 6th intercostal space at the anterior axillary line into the pleural cavity, with care to avoid placement into lung parenchyma or chest wall. The associated CSF effusion and iatrogenic pneumothorax resolve conservatively in almost all patients. Ventriculoatrial shunts are rarely performed because of complications of cor pulmonale, shunt nephritis, and catheter embolization. They require intraoperative ultrasound or fluoroscopy to document catheter placement into the atria via the internal jugular vein. We do not recommend their use.

Endoscopic third ventriculostomy: The morbidity of the life-long shunt has created interest in the use of endoscopic third ventriculostomy (ETV) a procedure that can effectively treat hydrocephalus without insertion of any foreign 
body. The principles of placement of an ETV include: frontal access, ventricular cannulation, insertion of a rigid or flexible neuroendoscope into the 3rd ventricle via the lateral ventricle and the foramen of Monroe. A fenestration is made in the base of the third ventricle between the infundibular recess and the mamillary bodies. This is commonly performed using a combination of electrocautery and a balloon dilator, thus creating a cerebrospinal fluid fistula between the subarachnoid space and the $3^{\text {rd }}$ ventricle (7). More recently, the concurrent performance of cauterization of the choroid plexus has been introduced as means of increasing shunt avoidance.

Classically, ETV was used for older children or adults with congenital aqueductal stenosis. Working in Uganda, Dr. Benjamin Warf has convincingly demonstrated however that ETV is a reasonable option for all children greater than 1 year of age (irrespective of hydrocephalus etiology) with a shunt avoidance rate of $80 \%$. In addition, with the use of cranial US and direct endoscopic visualization of the aqueduct, he stratified younger children based on aqueductal patency, and has demonstrated $70 \%$ success in those $<1$ year of age who have a post-infectious obstruction of the aqueduct. In addition, the use of ETV in combination with choroid plexus cauterizationhasincreased shuntavoidancefrom 35 to $76 \%$ in children with myelomeningocele, and from $20 \%$ to $71 \%$ in children $<1$ year of age with a non-post-infectious hydrocephalus and an open aqueduct (1).

Performance of ETV is beyond the scope of this review, and requires adequate mentoring by an experienced surgical team and significant technicalsupportfor maintaining the endoscopic system. Although the avoidance of a shunt (and the accompanying morbidity and mortality of shunt failure) in the developing world is a reasonable goal, the additional safety of ETV has not yet been proven in the long term, and most authors counsel that the same life-long follow-up is required in either case (2).

\section{Postoperative Complications}

Post-operative complications of shunt insertion can generally be classified as mechanical or infectious.
Mechanical shunt failure: Mechanical shunt failure can occur through proximal obstruction, distal obstruction, component separation/fracture/ migration or excessive CSF drainage (2).

Proximal or distal obstruction generally presents with signs and symptoms of increased ICP, whereas infectious failure presents with fever, redness or swelling at the surgical site, drainage of pus or CSF from the wound, nuchal rigidity, abdominal pain or peritonitis. Shunt obstruction in older patients often presents with headache, and is not associated with pain along the shunt tract (2). Although the signs and symptoms of shunt failure have been examined empirically, there is no ideal diagnostic test (2). Importantly, the opinion of parents is of greater diagnostic accuracy than that of referring clinicians (2): thus caregivers' opinions of their child's shunt function should not be dismissed lightly. In addition, children tend to present with similar manifestations during episodes of repeated shunt failure (13). Revision of a non-infected, but obstructed shunt should generally be approached from the cranial incision as obstruction is more common at the ventricular limb, and both ventricular and abdominal limbs can be tested in sequence from this location after they have been disconnected from the valve. Non-functioning ventricular catheters that are adherent can be removed by gentle traction under most circumstances, but we do not advocate more aggressive measures: rather, a new shunt should be placed at an alternative site.

Passage of a new peritoneal shunt down an established fibrous tract is occasionally possible as long as shunt infection has been ruled out preoperatively. Routine CSF culture in the absence of infectious signs or symptoms is not recommended as positive cultures in this setting are not predictive of subsequent infection (14).

Infectious shunt failure: Shunt infection is widely believed to be caused by contamination at the time of surgery, with occasional infections caused by later wound breakdown either due to CSF fistulisation or skin breakdown over hardware (2). Most shunt infections usually present within 3 months of shunt insertion, and almost all within six months (2). The rate of shunt infection in North America is 
approximately $8-10 \%$ (15) with some series reporting less than $1 \%$ (16), but in Africa is likely higher (17). The most common organisms grown are consistently S. epidermidis ( $40 \%)$, followed by $S$. aureus ( 20\%) (18).

Risk factors for shunt infection include prematurity $(19,20)$, elaborate shunt configurations, multiple separate shunts, previous shunt infection, surgical inexperience, myelomeningocele, post-operative CSF leak, and longer duration of surgery (21).

Common techniques to avoid shunt infection include the use of generous skin preparation, meticulous and consistent surgical technique, and preoperative prophylactic antibiotics (22). Although there is significant world-wide heterogeneity with regards to choice of antibiotic (23), we recommend ceftriaxone or cefazolin upon induction of anaesthesia. A Cochrane review in 2006 has supported the use of antibiotic prophylaxis in reducing shunt infection, with no evidence of benefit beyond 24 hours (24). As advocated by Faillace et al., the avoidance of shunt-to-skin contact resulted in a three-fold decrease in shunt infection rates from 9.1 to $2.9 \%$ (25). As mentioned earlier, valve design does not appear to have any effect on shunt infection. The surgeon should develop a consistent routine using the same equipment, and a meticulous approach: some authors have reported very low infection rates using similar techniques (26). Finally, shunts should not be relegated to the most inexperienced member of the team. We agree with the author who suggested: "Although less glamorous than other neurosurgical cases, shunting procedures deserve no less attention to detail" (27).

Because conventional antimicrobial techniques are not effective in treating the bacterial biofilms that affect most neurosurgicalrelated device infections, all infected shunts should be removed (28). This is combined with the insertion of an external ventricular drain with ongoing systemic and optional intrathecal antibiotics, with replacement of the shunt 10-14 days later with preoperative confirmation of a sterile CSF. Treatment with antibiotics alone has a high failure rate, and is probably only relevant in the high-risk surgical patient. Vancomycin has poor CSF penetration by IV route, and thus preservative-free ventricular vancomycin has been commonly used to treat shunt infections despite the incomplete understanding of side effects and toxicity (29).

Shuntalgia syndrome: This unusual shunt complication presents with focal discomfort around the shunt site without swelling, fluctuance or redness. There may be tenderness along the shunt itself, and there is usually a hard fibrotic sheath of scar tissue around the shunt. It is common in adolescents, and should generally be treated with conservative measures although narcotic analgesics have been required in some cases (2).

Shunt separation/fracture/migration: Although this occasionally occurs within the first few months of surgery it is thankfully rare. Clinical examination and if necessary a shunt series of plain radiographs along the entire shunt tract is sufficient to confirm the diagnosis. The phenomenon of arrested hydrocephalus (whereby a child develops true shunt independence) is rare: $80 \%$ of children who have stable ventricles despite a disconnected shunt have a raised ICP (30). Therefore most nonfunctioning shunts should be revised.

Hollow viscus perforation: Perforation of virtually every hollow viscus by the peritoneal catheter has been described, but is usually diagnosed by observation of the catheter protruding from the anus. The risk of hollow viscus perforation has been estimated at $1 / 1000$ shunt years (31). Remarkably, peritonitis is rarely a presenting feature, likely due to the gradual erosion of the shunt through bowel. Treatment is similar to other cases of shunt infection and involves removal of the shunt in its entirety via a single valve-site incision in the cranium, external drainage for 10-14 days with intrathecal or IV antibiotics followed by shunt replacement. Laparotomy is reserved for the rare case of peritonitis, but is not routinely required to remove the shunt.

\section{Prognosis and Outcomes}

The mortality associated with shunt placement is about $0.1 \%$, but shunt failure is more lethal at $1-4 \%$-especially in the African setting with its frequent difficult and delayed access to 
health care. The operative risk of ETV is still being defined, but perioperative mortality may approach 1\% (2). Shunts fail due to mechanical or infectious causes at a rate of $30-40 \%$ within the first year after placement (13), 15\% within the 2nd year, and $1-7 \%$ per year thereafter (2). Shunt infection is associated with reduced IQ and school performance, and a higher risk of future shunt infection (32). Although the risk of ventriculitis is reduced after ETV, it is not zero.

\section{Prevention}

In East Africa, the single most common cause of hydrocephalus is infection of the CNS, usually via neonatal meningitis or ventriculitis. Neonatal sepsis is common and is exacerbated by the lack of skilled perinatal care for the majority of births in Africa. Newborns presenting with febrile illness should ideally receive appropriate diagnostic tests and directed antibiotic therapy, and not just empiric therapy for presumed malaria. Efforts at improving perinatal care in developing countries will undoubtedly help to reduce the incidence of post-infectious hydrocephalus. The association of neural tube defects with hydrocephalus also mandates their prevention. There is excellent evidence to counsel for the use of $0.4 \mathrm{mg}$ of folic acid daily in all women of child-bearing age, and $4 \mathrm{mg}$ daily for mothers with previous children born with neural tube defects as a means of prevention (33). The fortification of wheat flour with folate has been met with success in many developed countries $(34,35)$ and there is increasing advocacy for it in the developing world (36).

\section{Ethical Issues}

There is a paucity of literature concerning the ethics of non-treatment of hydrocephalus, and issues surrounding resource allocation in the treatment of this disease. Informed consent of the patient and family should address the need for life-long ongoing surveillance of any child treated for hydrocephalus, the risk of shunt infection, shunt failure, and death. Families should be educated regarding signs of infection or shunt failure at the first admission.
Although we are not aware of any guidelines regarding assessment of medical futility in the setting of hydrocephalus, we recommend extreme caution in shunting children with head circumferences $>60 \mathrm{~cm}$, or in patients who have non-healing pressure sores on the cranium. For many of these children, aggressive surgical intervention is futile, and family resources are challenged by lengthy or repeated admissions.

Lastly, because of the higher incidence of hydrocephalus in developing nations, it is likely that research efforts will continue to be directed in this area. As in any research setting, attention to a meticulous informed consent process is required, particularly for those who are more vulnerable because of poverty or lower education.

\section{Recommendations}

1. Early treatment of hydrocephalus with ventriculo-peritoneal shunts remains the best method of preventing life-long disability from increased intracranial pressure in Africa.

2. Clinical symptoms and signs and cranial ultrasound are sufficient for the diagnosis and management of children with hydrocephalus.

3. Simple valved shunts such as the Chhabra ${ }^{\circledR}$ shunt are as effective as more sophisticated devices and save significant resources.

4. Shunt placement must be a thoroughly sterile procedure performed by skilled, experienced surgeons.

5. Both mechanical and infectious shunt complications can be significantly reduced through meticulous technique and experience.

6. Children shunted for hydrocephalus must be followed up for life and have rapid access to health care facilities in the case of complications.

7. Endoscopic third ventriculostomy shows significant promise for avoiding shunt morbidity in children with hydrocephalus, but remains limited by technology and skill.

8. Besides early diagnosis, prevention of hydrocephalus can be accomplished through efforts directed at the correct management of neonatal infections and folic acid supplementation for all women of child-bearing age. 


\section{References}

1. Warf B.C. Hydrocephalus in Uganda: The predominance of infectious origin and primary management with endoscopic third ventriculostomy. J. Neurosurg. (Pediatrics 1) 2005; 102: 1-15.

2. Garton H. and Piatt J. Hydrocephalus. J. Pediat. Clin. N.A. 2004; 51: 305-325.

3. Adeloye A. Management of infantile hydrocephalus in Central Africa. Tropical Doctor. 2001; 31: 67-70.

4. Heij H.A. The fate of ventricular peritoneal shunts and outcome of revision surgery. East Central Afr. J. Surg. 2001; 5: 17-19.

5. Saavedra-Lozano J., Booth T.N., Weprin B.E. and Ramilo O. Isolated cerebellar edema and obstructive hydrocephalus in a child with cerebral malaria. Pediat. Infect. Dis. J. 2001; 20(9): 908-911.

6. Iantosca M.R. and Drake J.M. Cerebrospinal fluid shunts. In: Albright A.L., Pollack I.F., Adelson P.D., eds. Operative techniques in pediatric Neurosurgery. New York: Thieme. 2000; 3-14.

7. Pople K. Hydrocephalus surgery (Oxford). 2004; 22(3): 60-63.

8. Carrion E., Hertzog J.H., Medlock M.D., et al. Use of acetazolamidetodecreasecerebrospinalfluid production in chronically ventilated patients with ventriculopleural shunts. Arch. Dis. Child. 2001; 84(1): 68-71.

9. Libenson M.H., Kaye E.M., Rosman N.P. et al. Acetazolamide and furosemide for posthemorrhagic hydrocephalus of the newborn. Pediatr. Neurol. 1999; 20(3): 185-191.

10. Kestle J., Drake J., Milner R., et al. Long-term follow-up data from the shunt design trial. Pediatr. Neurosurg. 2000; 33(5): 230-236.

11. Pollack I.F., Albright A.L. and Adelson P.D. A randomised, controlled study of a programmable shunt valve versus a conventional valve for patients with hydrocephalus. Neurosurg. 1999; 45: 1399-1411.

12. Warf B.C. Comparison of one-year outcomes for the Chhabra and Codman-Hakim micro precision shunt systems in Uganda: A prospective study in 195 children. J. Neurosurg. (Pediatrics 4).2005; 102: 358-362.

13. KestleJ. Pediatric hydrocephalus: current management. Neurol. Clin. N. Amer. 2003; 21(4): 883-895.

14. SteinbokP.,Cochrane D. and KestleJ.R. The significance of bacteriologically positive ventriculoperitoneal shunt components in the absence of other signs of shunt infection. J. Neurosurg. 1996; 85(5): 985-986.
15. Drake J.M., Kestle J.R., Milner R., et al. Randomised trial of cerebrospinal fluid shunt valve design in pediatric hydrocephalus. Neurosurg. 1998; 43: 294-305.

16. Choux M., Genitori L., Lang D., et al. Shunt implantation: Reducing the incidence of shunt infection. J. Neurosurg. 1992; 77: 875-880.

17. Mwang'ombe N.J. and Omulo T. Ventriculoperitoneal shunt surgery and shunt infections in children with non-tumour hydrocephalus at the Kenyatta National Hospital, Nairobi. East Afr. Med. J. 2000; 77(7): 386-390.

18. Turgut M., Alabaz D., Erbey F., et al. Cerebrospinal fluid shunt infections in children. Pediatr. Neurosurg. 2005; 41: 131-136.

19. Bruinsma N., Stobberingh E.E., Herpers M.J., et al. Subcutaneous ventricular catheter reservoir and ventriculoperitoneal drain-related infections in preterm infants and young children. Clin. Microbiol. Infect. 2000; 6(4): 202-206.

20. Kulkarni A.V., Drake J.M., Lambert-Pascrili M., et al. Cerebrospinal fluid shunt infection: A prospective study of risk factors. J. Neurosurg. 2001; 94: 195-201.

21. Kontny U., Höfling B., Gutjahr P., et al. CSF shunt infections in children. Infection. 1993; 21(2): 89-92.

22. Haines S.J. and Walters B.C. Antibiotic prophylaxis for cerebrospinal fluid shunts: A metanalysis. Neurosurg. 1994; 34(1): 89-92.

23. Biyani N., Grisaru-Soen G., Steinbok P., et al. Prophylactic antibiotics in paediatric shunt surgery. Childs. Nervous System. 2006; 22(11): 1465-1471.

24. Ratilal B., Costa J. and Sampaio C. Antibiotic prophylaxis for surgical introduction of intracranial ventricular shunts. Cochrane Database Syst. Rev. 2006; 3: CD005365.

25. Faillace W.J. A no-touch technique protocol to diminish cerebrospinal fluid shunt infection. Surg. Neurol. 1995; 43: 344-350.

26. Choksey M.S. and Malik I.A. Zero tolerance to shunt infections: Can it be achieved? J. Neurol. Neurosurg. Psychiat. 2004; 75(1): 87-91.

27. Kanev P.M. and Sheehan J.M. Reflections on shunt infection. Pediatr. Neurosurg. 2003; 39: 285-290.

28. Braxton E.E., Jr., Ehrlich G.D., Han-Stoodley P., et al. Role of biofilms in neurosurgical device-related infections. Neurosurg. Rev. 2005; 28(4): 249-255.

29. Bafeltowska J.J., Buzman E., Mandal K.M., et al. Therapeutic vancomycin monitoring in children with hydrocephalus during treatment of shunt infections. Surg. Neurol. 2004; 62(2): 142-150. 
30. Whittle I.R., Johnston H. and Besser M. Intracranial pressure changes in arrested hydrocephalus. J. Neurosurg. 1985; 62: 77-82.

31. Vinchon M., Baroncini M., Laurent T., et al. Bowel perforation caused by peritoneal shunt catheters: Diagnosis and treatment. Neurosurg. 2006; 58 (1 Suppl): ONS76-86.

32. Kanev P.M. and Park T.S. The treatment of hydrocephalus. Neurosurg. Clin. N. Amer. 1993; 4(4): 611619.

33. Laurence K.M., James N.E., Miller M.H., et al. Increased risk of recurrence of pregnancies complicated by fetal neural tube defects in mothers receiving poor diets and possible benefit of dietary counselling. Brit. Med. J.
1980; 281: 1592-1594.

34. Locksmith J.L. and Duff P. Preventing NTDs: The importance of periconceptional folic acid supplements. Obstet. Gynecol. 1998; 91(6): 1027-1034.

35. Bol K.A., Collins J.S. and Kirby R.S. Survival of infants with neural tube defects in the presence of folic acid fortification. Pediatrics. 2006; 117(3): 803-813.

36. http://www.gainhealth.org Global Alliance for Improved Nutrition.

37. Eichholzer M., Tcinz O. and Zimmermann R. Folic acid: a public health challenge. Lancet. 2006; 22; 367(9519): 1352-1361. 